\title{
Enfermedad oclusiva aortoilíaca o síndrome de Leriche
}

\author{
Juanita Martínez ${ }^{1}$, Jaime José DíaZ $Z^{2}$, Vanesa Paola Luján², María Rosario FernándeZ ${ }^{3}$, Efraín Ramírez ${ }^{4}$
}

Palabras clave: enfermedades de la aorta; arteria ilíaca; claudicación intermitente; enfermedad arterial periférica; oclusión vascular; síndrome de Leriche.

\section{Resumen}

La enfermedad oclusiva aortoiliaca, o sindrome de Leriche, es una forma de la enfermedad arterial periférica, en la cual existe una oclusión de las arterias ilíacas desde su bifurcación. Su manifestación clínica es variable y la claudicación intermitente es la más frecuente; no obstante, es una enfermedad usualmente subdiagnosticada por su cronicidad y el sedentarismo de los adultos mayores, quienes más la presentan. Para el diagnóstico se cuenta con un abanico de opciones, que incluyen pruebas invasivas y no invasivas; la arteriografia es el método de elección. El tratamiento, por su parte, involucra cambios en el estilo de vida aunados a un tratamiento conservador o quirúrgico, según cada individuo.

Médico, estudiante de posgrado de Cirugía General, Universidad del Sinú, Cartagena, Colombia

2 Médico, estudiante de posgrado de Cirugía General, Universidad de Cartagena, Cartagena, Colombia

3 Médico, Universidad de Cartagena, Cartagena, Colombia

4 Médico, cirujano vascular periférico; docente, Departamento Quirúrgico, Universidad de Cartagena y Universidad del Sinú, Cartagena, Colombia

Fecha de recibido: 16 de marzo de 2017

Fecha de aprobación: 1 de abril de 2017

Citar como: Martínez J, Díaz JJ, Luján VP, Fernández MR, Ramírez E. Enfermedad oclusiva aortoilíaca o síndrome de Leriche. Rev Colomb Cir. 2017;32:214-22.

\section{Introducción}

El síndrome de Leriche se define como una oclusión de las ramas principales de la aorta abdominal por debajo de la salida de las arterias renales, con compromiso de todo el árbol arterial, incluyendo las arterias ilíacas y femorales. Se caracteriza por signos clínicos tales como impotencia sexual, ausencia de pulso en las arterias femorales, debilidad y entumecimiento en la región lumbar, los glúteos, las caderas y las extremidades inferiores.

Fue descrita por primera vez en 1814 por Robert Graham (1786-1845), médico de la Royal Infirmary de Glasgow; sin embargo, la definición actual se debe al cirujano francés René Leriche quien la describió en 1940 como una oclusión de la aorta terminal y sus ramas por la presencia de trombos. Leriche vinculó la formación de trombos arteriales con una tríada de síntomas que incluye claudicación, impotencia y disminución de los pulsos periféricos. Desde entonces, el epónimo "síndrome de Leriche" ha sido utilizado para referirse a la enfermedad oclusiva aortoilíaca ${ }^{1}$.

La incidencia y la prevalencia exactas de esta enfermedad han sido difíciles de determinar, debido a que muchos pacientes que cursan con esta patología son asintomáticos, como consecuencia del desarrollo de redes vasculares colaterales ${ }^{2}$. Sin embargo, es una entidad que se ha relacionado fuertemente con la edad avanzada; se presenta con mayor frecuencia a partir de la sexta década de la vida. Afecta principalmente a pacientes del sexo masculino y con predisposición a la enfermedad arterial periférica, expuestos a factores de 
riesgo tales como tabaquismo, hipertensión arterial sistémica, dislipidemias, obesidad y diabetes ${ }^{3}$, en quienes las placas ateromatosas localizadas en la aorta infrarrenal y las arterias ilíacas causan síntomas de obstrucción por bajo flujo sanguíneo arterial.

Se han reportado casos en mujeres, en quienes se han relacionado otros trastornos asociados a las vasculitis, tales como lupus eritematoso sistémico y espondilitis anquilosante, entre otros ${ }^{4}$.

\section{Anatomía}

La aorta es la principal arteria del organismo, la cual se encarga de la irrigación de toda la economía corporal. Para entrar al abdomen, se introduce en el diafragma a la altura de T12, donde se convierte en una estructura retroperitoneal, que discurre dando sus ramas para los órganos intrabdominales; posteriormente, se posiciona ligeramente hacia la izquierda y se bifurca a la altura de L4 para dar origen a las arterias ilíacas comunes -derecha e izquierda-, que suministran el aporte sanguíneo a las vísceras pélvicas y a las extremidades inferiores. En su porción infrarrenal, la aorta presenta las mayores alteraciones en el flujo dinámico sanguíneo, lo cual la hace particularmente propensa a la formación de placas ateromatosas, las cuales son más frecuentes en su bifurcación, donde el ángulo formado por el vaso es un sitio usual de depósito ${ }^{5}$.

La localización anatómica de la lesión influye directamente en la vía colateral desarrollada, siendo su posible génesis a partir de la circulación sistémica o visceral; cada una de estas vías ayuda a mantener la perfusión de las extremidades inferiores. Las arterias viscerales colaterales son más frecuentes en lesiones distales a la aorta, incluyendo lesiones de la bifurcación aórtica o de las arterias ilíacas comunes (figura 1). Las colaterales provenientes de la circulación sistémica son más prominentes en las lesiones proximales de la aorta o en aquellas inmediatamente inferiores a las arterias renales (figura 2) ${ }^{5}$.

\section{Clasificación}

La localización anatómica de las lesiones ateromatosas repercute directamente en la clasificación de la enfermedad, así como en la elección de la modalidad de tratamiento.
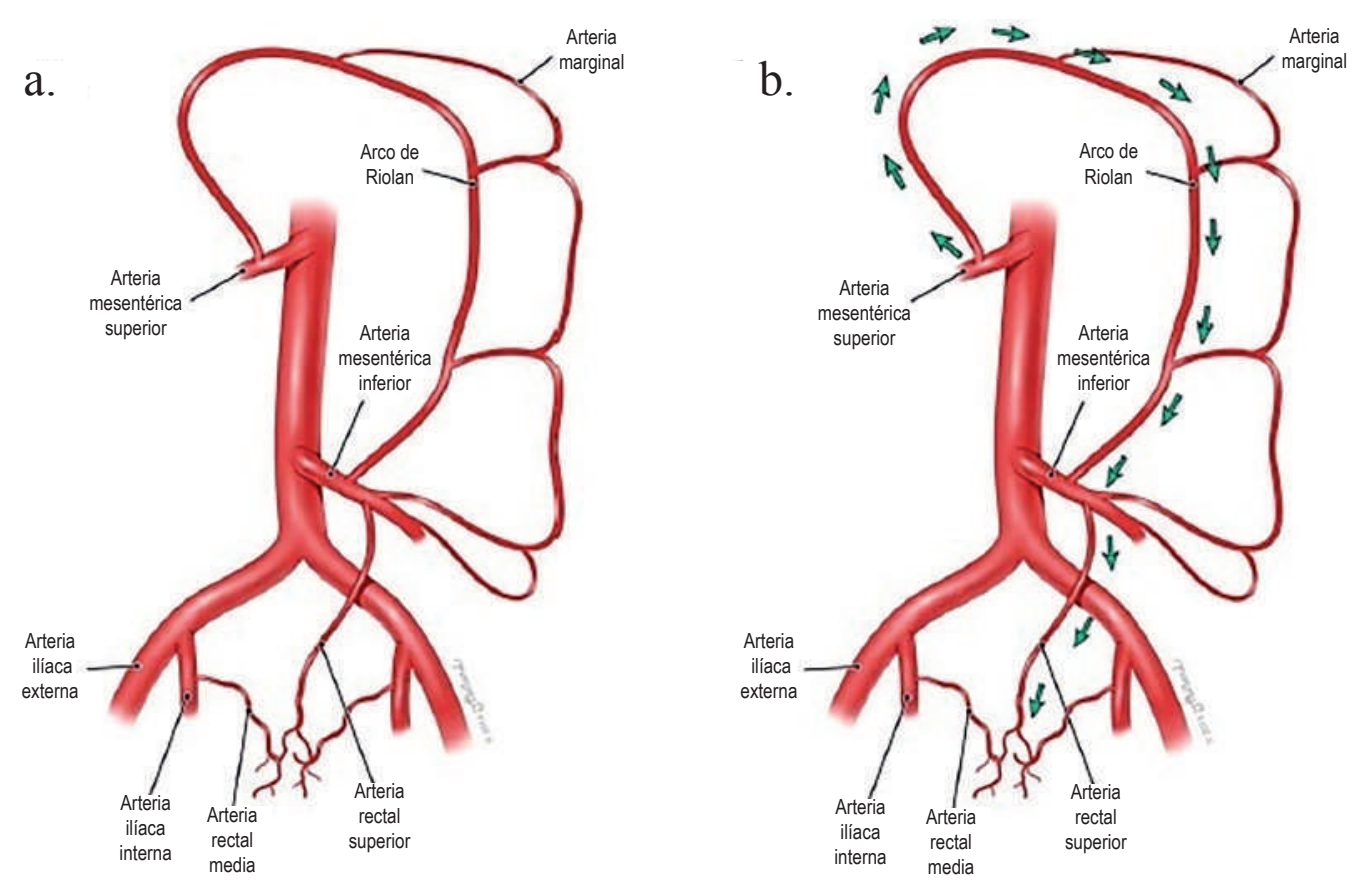

Figura 1. a. Vias de circulación visceral colateral, en oclusión aortoiliaca. b. Ramas de la arteria mesentérica superior; arco de Riolan; arteria marginal de Drumond; arteria rectal superior; arteria rectal media; arteria iliaca interna.

Reproducido y traducido con autorización de: Anatomical significance in aortoiliac occlusive disease. Clin Anat. 2014;27:1264-74. 


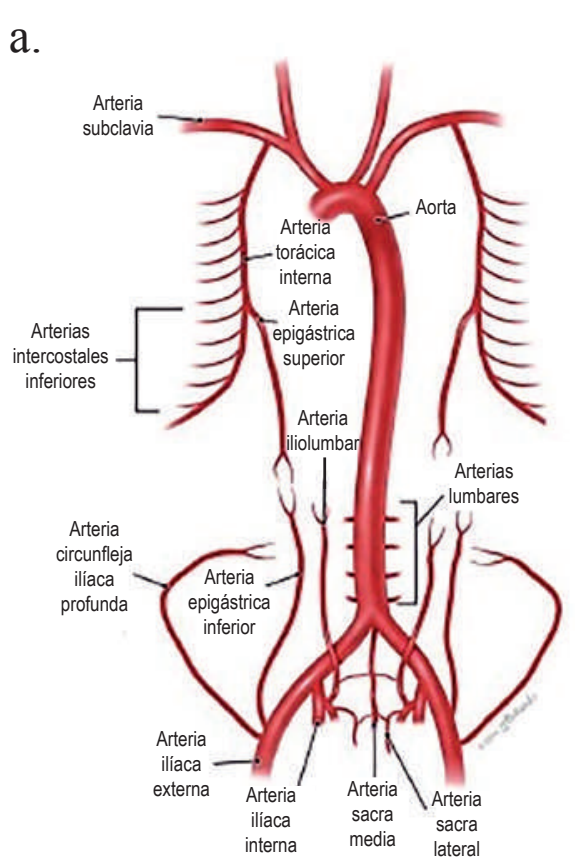

b.

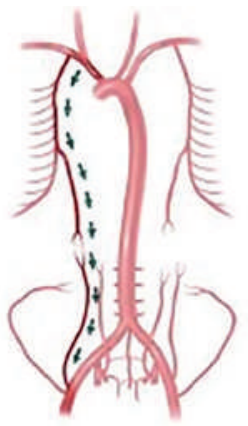

d.

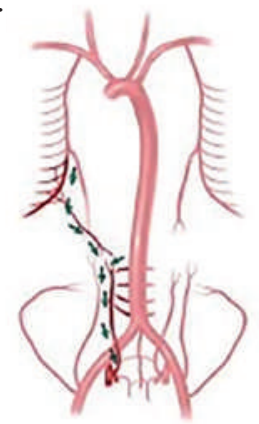

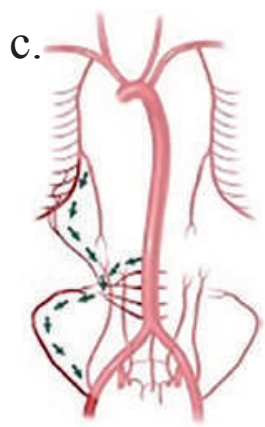

e.

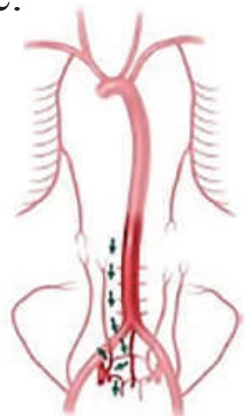

FiguRA 2. a. Vias colaterales sistémicas, en oclusión aortoilíaca. b. Arteria subclavia; arteria torácica interna; arteria epigástrica superior; arteria epigástrica inferior; arteria iliaca externa. c. arterias intercostales inferiores; arterias lumbares; ramo ascendente de la arteria circunfleja iliaca externa; arteria iliaca externa. d. Arterias intercostales inferiores, arterias lumbares; arteria iliolumbar; arteria iliaca interna. e. Aorta abdominal; arteria sacra media; arteria iliolumbar; arteria sacra lateral; arteria iliaca interna. Reproducido y traducido con autorización de: Anatomical significance in aortoiliac occlusive disease. Clin Anat. 2014;27:1264-74.

Con base en la localización de la lesión ateromatosa, se ha propuesto el siguiente sistema de clasificación ${ }^{5}$.

- Tipo I: involucra la aorta abdominal infrarrenal y las arterias ilíacas comunes.

- Tipo II: involucra la aorta abdominal infrarrenal, las arterias ilíacas comunes, las arterias ilíacas externas y la arteria femoral común.
- Tipo III: involucra la aorta abdominal infrarrenal, las arterias ilíacas comunes, las ilíacas externas, las femorales comunes, la bifurcación, las poplíteas, o las arterias tibiales.

Otro sistema de clasificación es el establecido por el consenso TASC II (Trans-Atlantic Inter-Society Consensus II), cuyos parámetros se basan en la forma y la distribución de las lesiones, además de la gravedad de la enfermedad ${ }^{3}$.

\section{Clasificación TASC II para enfermedad oclusiva aortoíliaca}

Tipo A

Estenosis unilateral o bilateral de la arteria ilíaca común, o estenosis unilateral o bilateral de la arteria ilíaca externa en un segmento de $3 \mathrm{~cm}$ o menos

Tipo B

Estenosis de $3 \mathrm{~cm}$ o menos de la aorta infrarrenal

- Oclusión unilateral de la arteria ilíaca común

- Estenosis simple o múltiple, de 3 a $10 \mathrm{~cm}$ en total, incluyendo la arteria ilíaca externa y sin extenderse a la arteria femoral común

- Estenosis unilateral de la arteria ilíaca común, sin incluir el origen de la arteria ilíaca interna o la femoral común
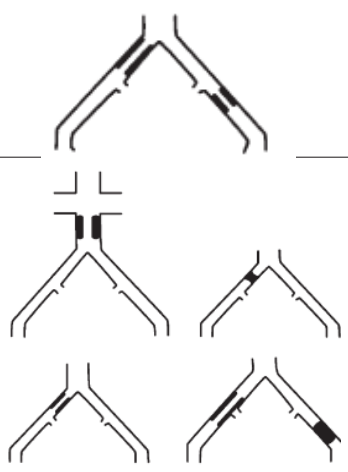
Tipo C

- Oclusión bilateral de ambas arterias ilíacas comunes

- Estenosis bilateral de las arterias ilíacas externas, de 3 a $10 \mathrm{~cm}$, sin extenderse dentro de la femoral común

- Estenosis unilateral de la arteria ilíaca externa, extendiéndose hasta la femoral común

- Oclusión unilateral de la arteria ilíaca externa que involucra el origen de la ilíaca interna, la arteria femoral común o ambas

- Calcificación unilateral de la arteria ilíaca externa y oclusión que involucra o no el origen de la arteria iliaca interna, la arteria femoral común o ambas

Tipo D

- Oclusión aortoilíaca infrarrenal

- Enfermedad difusa que involucra la aorta y ambas arterias iliacas

- Estenosis difusa múltiple que involucra la arteria ilíaca común unilateral, la ilíaca externa y la femoral común

- Oclusión unilateral de la arteria femoral común y la arteria ilíaca externa

- Oclusión bilateral de la arteria ilíaca externa

- Estenosis de la arteria ilíaca común, en pacientes con aneurisma de la aorta abdominal

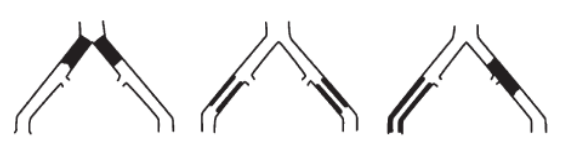

\section{1}


crítica de la extremidad, factor fuertemente asociado con gran morbimortalidad ${ }^{7}$.

En los hombres, el síndrome puede cursar con disfunción eréctil por reducción del flujo sanguíneo en la arteria ilíaca interna, que se traduce en una disminución del riego sanguíneo en los espacios sinusoidales del pene. Esta manifestación puede encontrarse en lesiones proximales a la bifurcación de la arteria iliaca común, en las cuales se ve afectada la perfusión por las ramas responsables de iniciar y mantener una erección: la arteria pudenda o arteria dorsal del pene ${ }^{8}$.

Se ha reportado la presentación de oclusión aortoilíaca con síntomas atípicos, como neuropatía, síndrome de cauda equina ${ }^{9}$, o en algunos casos, como una catástrofe isquémica que involucra la irrigación de órganos abdominales y los miembros inferiores ${ }^{10}$.

\section{Diagnóstico}

En la mayoría de los pacientes, los antecedentes médicos, una adecuada anamnesis y un examen físico preciso, pueden establecer o excluir el diagnóstico de la enfermedad. Como pruebas confirmatorias, se cuenta con una amplia variedad de estudios invasivos y no invasivos, cada uno de los cuales presenta ventajas y desventajas ${ }^{11}$.

1. Estudios vasculares no invasivos. Su objetivo es confirmar la existencia de lesiones, su localización, repercusión hemodinámica y el estado del lecho arterial distal ${ }^{12}$. Se dispone de los siguientes métodos:

- Índice tobillo-brazo: es una herramienta diagnóstica clínica fundamental en el estudio de la enfermedad arterial de los miembros inferiores, con sensibilidad de $79 \%$ y especificidad de $86 \%$; el punto de corte es de 0,9 y los valores por debajo de este son anormales (recomendación IB).

- Ultrasonido Doppler continuo y pulsado: permite evaluar la presencia o ausencia de flujo sanguíneo y sus características hemodinámicas (laminar, turbulento, velocidad de flujo, resistencias distales) en cada uno de los vasos de las extremidades. Las formas de la onda Doppler de la arteria femoral común se han utilizado para el diagnóstico de un trastorno de entrada. La onda normal es trifásica con un componente negativo posterior a la sístole.
En una obstrucción aortoilíaca se observa pérdida relativamente temprana de esta onda y la presencia de una configuración bifásica, amortiguada, lo que indica una alteración del flujo.

- Ecografia Doppler: aporta información morfológica de las estructuras vasculares (calcificaciones de la pared arterial, placas de ateroma, ulceraciones, trombos en la luz arterial) y las alteraciones hemodinámicas consecuentes. Cuenta con una sensibilidad de 85 a $90 \%$ y especificidad mayor de $95 \%$. Es el estudio de primera línea para confirmar y localizar la enfermedad arterial de los miembros inferiores (recomendación IB).

- Presiones segmentarias: permiten determinar la presión de la sangre en cada sector de la extremidad (muslo, pierna y tobillo), correlacionándolas entre sí con la extremidad del lado opuesto y con la presión en antebrazo (considerada como normal). Puede tener una desventaja debido a que el manguito arterial en el muslo proximal no necesariamente indica la presión del sistema ilíaco o del femoral común, debido a que no se coloca lo suficientemente alto para ocluir estos vasos. Así, una baja presión en el muslo proximal puede representar oclusión de la arteria femoral superficial y de la femoral profunda, más que una alteración del flujo en el sistema aortoilíaco ${ }^{13}$.

- Angiotomografía computarizada (angio-TC) y angiorresonancia: constituyen técnicas que permiten una adecuada caracterización de las arterias periféricas. La angio-TC presenta sensibilidad y especificidad mayores de $95 \%$ para diagnósticos de estenosis u oclusión de los segmentos aortoilíacos y femoropoplíteos, con menor rendimiento en los segmentos infrapatelares; mientras que la angiorresonancia ofrece una sensibilidad de $100 \%$ y una especificidad de $84 \%$ para la porción infrapatelar, siendo mayor al $90 \%$ en los segmentos más proximales. Se debe tener en cuenta la función renal para el uso de medio de contraste no iónico o de gadolinio, por el riesgo de nefrotoxicidad ${ }^{11}$.

- Otros métodos de menor valor diagnóstico son la pletismografía, la presión parcial de oxígeno $\left(\mathrm{PO}_{2}\right.$ transcutánea) y la capilaroscopia.

2. Exploraciones invasivas. La arteriografía, en sus diversas modalidades, es el método estándar para el 
diagnóstico de la enfermedad oclusiva aortoilíaca, y permite evaluar la extensión, la topografía de las lesiones y el desarrollo de vasos colaterales. Con esta se observa la morfología desde las arterias renales hasta los pies, y se puede estimar la presencia de una afección concurrente de los vasos mesentéricos inferiores, hipogástricos y femorales profundos. Por seguridad, fácil acceso y menores complicaciones, se prefiere realizarla por acceso arterial transfemoral; cuando los pulsos femorales no son palpables, se puede abordar por un acceso arterial radial, cubital, braquial o translumbar. Es un estudio muy útil para hacer el diagnóstico diferencial con otros procesos que cursan con isquemia aguda, especialmente con las embolias arteriales, y es un método ideal para planificar la estrategia quirúrgica ${ }^{12}$.

\section{Tratamiento}

El manejo de la enfermedad oclusiva aortoilíaca, en el contexto de una variante de la enfermedad arterial periférica, debe incluir cambios en el estilo de vida y la prevención de los factores de riesgo modificables, así como tratamiento farmacológico, con énfasis en el control del síntoma cardinal de esta entidad, la claudicación intermitente ${ }^{11}$.

El ejercicio físico supervisado ha demostrado mejoría de los síntomas, y aumento de la velocidad, distancia y duración de la deambulación. Siempre que no esté contraindicado, debe estar incluido en el programa de manejo del paciente con enfermedad oclusiva aortoilíaca (recomendación IA).

Desde el punto de vista farmacológico, el cilostazol es el tratamiento de elección de la claudicación intermitente (recomendación IA). Este es un inhibidor específico de la fosfodiesterasa III celular que, mediante su efecto antitrombótico permite el manejo de los síntomas de la enfermedad. De igual manera, el naftidrofurilo, el cual es un vasodilatador cerebral y periférico cuyo mecanismo de acción está relacionado con el bloqueo de los receptores serotoninérgicos (5HT2) localizados en el músculo liso vascular, estimula el metabolismo aerobio intracelular, reduce los niveles de ácido láctico y aumenta las concentraciones de ATP, con la consecuente protección celular de la isquemia vascular. Además de los dos anteriores, debe incluirse el tratamiento antiagregante plaquetario con ácido acetil-salicílico (ASA) (recomendación IB), o con clopidogrel, en los pacientes con contraindicación para el ácido acetil-salicílico (recomendación IIA). Las estatinas deben usarse sin tener en cuenta los niveles de colesterol sérico, ya que se ha demostrado prevención de la progresión de la enfermedad, y reducción de la morbilidad cardiopulmonar y cerebrovascular (recomendación IB) ${ }^{14-16}$.

Los inhibidores de la enzima convertidora de angiotensina, los betabloqueadores, la pentoxifilina y otros, no han demostrado repercusiones significativas en la morbimortalidad de esta entidad. Existen otras alternativas para el tratamiento médico de la enfermedad arterial periférica grave, como los prostanoides, en especial, la prostaglandina $\mathrm{E}$, para la claudicación intermitente, como coadyuvante en la reperfusión endovascular o a cielo abierto, y para tratar la claudicación cuando no son posibles la reperfusión o la cicatrización de úlceras de origen arterial ${ }^{17,18}$.

Por otra parte, la terapia con células madres ha demostrado ser una alternativa factible, relativamente segura y efectiva en pacientes con enfermedad arterial periférica no aptos para la reperfusión, con mejoría del índice tobillo-brazo, reducción del dolor y disminución de las tasas de amputación ${ }^{19,20}$.

El tratamiento quirúrgico de la enfermedad oclusiva aortoilíaca ha evolucionado en los últimos años, desde el manejo a cielo abierto hasta el endovascular. Este último es la opción preferida en las lesiones más simples (TASC A, B e incluso C) y dejando la cirugía abierta para los casos complejos (TASC C, D), en los que no sea posible el abordaje mínimamente invasivo; además en un tratamiento de segunda línea o complementario en los casos que requieren manejo híbrido, es decir, cirugía abierta y endovascular en el mismo procedimiento ${ }^{3,21}$.

Las principales indicaciones para la reconstrucción aortoilíaca son el riesgo de pérdida de la extremidad, con dolor de reposo, ulceración o gangrena, y fenómenos ateroembólicos, como el síndrome de dedos azules de los pies. La presencia de claudicación se considera un signo de gravedad suficiente para restringir la calidad de vida del paciente, por lo que es una indicación aceptable para practicar procedimientos de reconstrucción aortoilíaca en personas que no tengan contraindicaciones. 
El manejo a cielo abierto de la enfermedad oclusiva aortoilíaca incluye varias opciones de tratamiento quirúrgico que permiten al cirujano tratante la elección de uno u otro de acuerdo con el estado clínico del paciente. La edad y las enfermedades concomitantes son factores primordiales que se deben tener en cuenta para decidir sobre el procedimiento quirúrgico. García, et al., consideran el uso de escalas pronósticas para valorar el riesgo y predecir resultados a largo plazo en pacientes sometidos a derivación (bypass) aortofemoral. Los índices de comorbilidad de Charlson ajustados a la edad y la escala de aneurisma de Glasgow, tienen impacto como factor predictivo para la morbilidad y la supervivencia en pacientes candidatos para derivación aortofemoral, y permiten evaluar los pacientes de alto riesgo, quienes se convierten en candidatos para el tratamiento endovascular o la derivación extraanatómica ${ }^{22}$.

\section{Manejo a cielo abierto}

Entre las opciones para tratamiento quirúrgico a cielo abierto, se cuenta con la derivación aortofemoral, la derivación iliofemoral y la endarterectomía aortoilíaca, y su elección depende de la clasificación TASC. En una amplia revisión bibliográfica, Chiu, et al., compararon los resultados y la efectividad de cada procedimiento, demostrando que la endarterectomía aortoilíaca cuenta con tasas más bajas de morbilidad y mortalidad perioperatoria; por otra parte, en cuanto a la efectividad a largo plazo, no hubo diferencias significativas entre estos procedimientos ${ }^{23}$.

En cuanto a la vía de abordaje en los procedimientos quirúrgicos para el tratamiento de la enfermedad oclusiva aortoilíaca, se ha aceptado amplia y tradicionalmente el abordaje transperitoneal como la técnica de elección en estas cirugías, sobre el retroperitoneal, teniendo en cuenta su simplicidad y rapidez para acceder a las estructuras vasculares en la cavidad abdominal; sin embargo, existen estudios aislados, como el de Muhamed, et al., del centro clínico de la Universidad de Sarajevo, en los cuales se concluye que el abordaje retroperitoneal ofrece mejores resultados como menor tiempo quirúrgico, menor incidencia de íleo paralítico, disminución del tiempo de hospitalización en la unidad de cuidados intensivos y en salas generales, y consecuente disminución en los $\operatorname{costos}^{24}$.

Las intervenciones extraanatómicas, como la derivación axilo-unifemoral, la axilo-bifemoral o la torácico- bifemoral, permanecen como última opción terapéutica en pacientes que no son candidatos para reperfusión anatómica abierta o endovascular ${ }^{11}$.

\section{Tratamiento endovascular}

El tratamiento endovascular ha crecido como herramienta terapéutica para la enfermedad oclusiva aortoilíaca. Por recomendaciones del consenso TASC II, las lesiones de tipo A y B deben manejarse de forma endovascular como primera elección, con buenos resultados posquirúrgicos; mientras que las lesiones de tipo $\mathrm{C}$ y $\mathrm{D}$ tienen un mayor índice de fallas con este manejo.

Se emplea el abordaje endovascular como primera línea en pacientes con múltiples enfermedades concomitantes y alto riesgo de complicaciones con la intervención a cielo abierto. Algunas series de casos, como la de Vikram, et al., en la cual se incluyeron pacientes con enfermedad oclusiva aortoilíaca con clasificación TASC C y D, han informado buenos resultados con el tratamiento endovascular, con tasas de permeabilidad vascular de $76 \%$ a tres años en la primera intervención, de $90 \%$ después de la segunda intervención, y de $97 \%$ de salvamento de la extremidad ${ }^{3,25}$.

Entre las opciones terapéuticas se cuenta con la angioplastia con balón para la dilatación de trayectos estenóticos y con las endoprótesis (stents) vasculares. En una revisión bibliográfica que incluyó 1.711 pacientes, Wisselink, et al., concluyeron que el tratamiento endovascular es exitoso teniendo en cuenta la experiencia o habilidad del equipo de trabajo y una selección adecuada de los pacientes candidatos, con tasas de mejoría clínica de 83 a $100 \%$, mortalidad menor de $7 \%$, y tasas de complicaciones con incidencia variable de 3 a $45 \%$, incluyendo embolia distal, hematoma en el sitio de punción, seudoaneurismas, ruptura arterial y disección arterial, no obstante, con amplias posibilidades de resolución por la misma vía, logrando una permeabilidad vascular en el primer año de 60 a $86 \%$ y, después de una segunda intervención, de 80 a $98 \%$, igualando los resultados de la cirugía abierta ${ }^{26}$.

\section{Abordaje a cielo abierto Vs. endovascular}

Existen ventajas y desventajas para los dos grupos de procedimientos quirúrgicos en el tratamiento de la enfermedad oclusiva aortoilíaca, a cielo abierto y 
endovasculares. Los procedimientos a cielo abierto muestran mejores resultados en cuanto a la permeabilidad vascular y la durabilidad de la reperfusión a largo plazo, mientras que los procedimientos endovasculares tienen una menor morbilidad, con menor tiempo quirúrgico y estancia hospitalaria.

En un metaanálisis que incluyó 5.358 pacientes, entre 1989 y 2010, Indes, et al., compararon los resultados entre el tratamiento con derivación a cielo abierto y con derivación endovascular, encontrando una estancia hospitalaria mayor en los pacientes con reperfusión por cirugía abierta ( 13 Vs. 4 días; $\mathrm{p}<0,001)$; de igual manera, los pacientes manejados con derivación a cielo abierto presentaron más complicaciones (18 Vs. $13,4 \% ; \mathrm{p}<0,001)$ y mayor mortalidad a 30 días $(2,6$ Vs. $0,7 \% ; \mathrm{p}<0,001)$. Con respecto a las tasas de permeabilidad primaria y secundaria en el seguimiento a uno, tres y cinco años, fueron mayores para el grupo de derivación a cielo abierto que para el grupo con tratamiento endovascular, con adecuada significancia estadística. De esta manera, concluyeron que la permeabilidad y durabilidad de la reperfusión con la técnica abierta son superiores que con la endovascular, con seguimientos a uno, tres y cinco años $(\mathrm{p}<0,001)$, pese a tener una mayor estancia hospitalaria y riesgo de morbimortalidad ${ }^{27}$.

\section{Conclusión}

La enfermedad oclusiva aortoilíaca, o síndrome de Leriche, es una entidad subdiagnosticada, teniendo en cuenta el gran número de pacientes asintomáticos debido al sedentarismo y al desarrollo de circulación colateral en el contexto de una enfermedad crónica. La claudicación intermitente es su síntoma principal y su morbilidad es elevada, dado el compromiso anatómico de los miembros inferiores que genera y su impacto incapacitante en la calidad de vida de los pacientes.

Existen numerosas herramientas para el diagnóstico, siempre teniendo como pilar la sospecha clínica; en cuanto a su tratamiento, el endovascular se consolida como el tratamiento de primera línea por sus menores tasas de morbimortalidad; sin embargo, la cirugía a cielo abierto anatómica o extraanatómica seguirá siendo una opción terapéutica de acuerdo con las características de la lesión y el estado clínico del paciente.

\title{
Aorto-iliac occlusive arterial disease or Leriche’s syndrome
}

\begin{abstract}
Aortoiliac occlusive disease (AIOD) or Leriche's syndrome is a form of peripheral arterial disease in which there is occlusion of the iliac arteries starting at the aorto- iliac bifurcation. Clinical manifestation are variable, with intermittent claudication being the most frequent; nevertheless, it is usually underdiagnosed because of its chronicity and the sedentarism of the elderly, the age group that is most frequently affected. A variety of options are available for the diagnosis, including invasive and non-invasive tests, arteriography being the method of choice. Treatment involves changes in the lifestyle coupled with a conservative or surgical management depending on the individual patient.
\end{abstract}

Key words: Aortic diseases; iliac artery; intermittent claudication; peripheral arterial disease; vascular occlusion; Leriche's syndrome.

\section{Referencias}

1. Ioncioaia B, Bud IT, Muresan M. René leriche and the development of 20th century surgery. Clujul Medical. 2016;89:176-80.

2. Morotti A, Busso M, Cinardo P, Bonomo K, Angelino V, Cardinale L, et al. When collateral vessels matter: Asymptomatic Leriche syndrome. Clin Case Rep. 2015;3:960-1.

3. Norgren L, Hiatt WR, Dormandy JA, Nehler MR, Harris KA, Fowkes FGR, Tasc II Working Group, et al. Inter-society consensus for the management of peripheral arterial disease (TASC II). Eur J Vasc EndovascSurg. 2007;33(Supl.1):S1-75.
4. Higashihara T, Shiode N, Kawase T, Tamekiyo H, Otsuka M, Okimoto T, et al. Endovascular therapy is effective for Leriche syndrome with deep vein thrombosis. Case Rep Cardiol. 2015;2015:395205.

5. Wooten C, Hayat M, Plessis M, Cesmebasi A, Koesterer M, Daly KP, et al. Anatomical significance in aortoiliac occlusive disease. Clin Anat. 2014;27:1264-74.

6. Frederick M, Newman J, Kohlwes J. Leriche syndrome. J Gen Intern Med. 2010;25:1102-4. 
7. de Sobregrau RC, Viver E, Rodríguez-Mori E, Jiménez-Cossio JA, Castromil E, Barreiro A. Estudio clínico y tratamiento quirúrgico en el Síndrome de Leriche. Angiología. 1975;27(3): 131-39

8. Bhatia MS, Gautam P, Saha R. Leriche syndrome presenting as depression with erectile dysfunction. J Clin Diagn Res. 2016;10:VD01-2.

9. Groth M, Much C, Fiehler J. Manifestation of acute Leriche syndrome as cauda equina syndrome. Rofo. 2013;185:375-6.

10. McCoy CE, Patierno S, Lotfipour S. Leriche syndrome presenting with multisystem vaso-occlusive catastrophe. West J Emerg Med. 2015;16:583-6.

11. Sociedad Argentina de Cardiología. Consenso de enfermedad vascular periférica. Rev Argent Cardiol. 2015;83(Supl.3). Fecha de consulta: 2 de febrero de 2017. Disponible en: http:// www.sac.org.ar/wp-content/uploads/2016/01/consenso-deenfermedad-vascular-periferica.pdf .

12. Takigawa M, Akutsu K, Kasai S, Tamori Y, Yoshimuta T, Higashi $\mathrm{M}$, et al. Angiographic documentation of aortoiliac occlusion in Leriche's syndrome. Can J Cardiol. 2008;24:568.

13. Brunicardi FC, Andersen D, Billiar T, Dunn D, Hunter J, Matthews J, et al. Schwartz. Principios de cirugía. Novena edición. México, D.F.: McGraw-Hill; 2010.

14. Meng Y, Squires H, Stevens JW, Simpson E, Harnan S, Thomas $\mathrm{S}$, et al. Cost-effectiveness of cilostazol, naftidrofuryl oxalate, and pentoxifylline for the treatment of intermittent claudication in people with peripheral arterial disease. Angiology. 2014;65:190-7.

15. Jeng JS, Sun Y, Lee JT, Lin RT, Chen CH, Po HL, et al. The efficacy and safety of cilostazol in ischemic stroke patients with peripheral arterial disease (SPAD): Protocol of a randomized, double-blind, placebo-controlled multicenter trial. Int J Stroke. 2015;10:123-7.

16. Stevens JW, Simpson E, Harnan S, Squires H, Meng Y, Thomas S, et al. Systematic review of the efficacy of cilostazol, naftidrofuryl oxalate and pentoxifylline for the treatment of intermittent claudication. Br J Surg. 2012;99:1630-8.

17. Vitale V, Monami M, Mannucci E. Prostanoids in patients with peripheral arterial disease. A meta-analysis of placebocontrolled randomized clinical trials. J Diabetes Complications. 2016;30:161-6.

18. Lawall H, Pokrovsky A, Checinski P, Ratushnyuk A, Hamm G, Randerath $\mathrm{O}$, et al. Efficacy and safety of alprostadil in patients with peripheral arterial occlusive disease fontaine stage IV: Results of a placebo controlled randomised multicentre trial (especial). Eur J Vasc Endovasc Surg. 2017;53:559-66

19. Fadini GP, Agostini C, Avogaro A. Autologous stem cell therapy for peripheral arterial disease. Meta-analysis and systematic review of the literature. Atherosclerosis. 2010;209:10-7.

20. Lawall H, Bramlage P, Amann B. Treatment of peripheral arterial disease using stem and progenitor cell therapy. J Vasc Surg. 2011;53:445-53.

21. Talledo O, Valdez LM, Torres L, De la Peña O, Calle A. Enfermedad oclusiva aorto-ilíaca: del tratamiento quirúrgico al endovascular. Revista Médica Herediana. 2015;26:177-85.

22. García F, Marchena J, Cabrera V, Hermida M, Sotgiu E. Evaluation of four risk-scoring methods to predict long-term outcomes in patients undergoing aorto-bifemoral bypass for aorto-iliac occlusive disease. Int J Angiol. 2012;21:19-28.

23. Chiu KW, Davies RS, Nightingale PG, Bradbury AW, Adam DJ. Review of direct anatomical open surgical management of atherosclerotic aorto-iliac occlusive disease. Eur J Vasc Endovasc Surg. 2010;39:460-71

24. Djedovic M, Rustempasic N, Djedovic S, Totic D, Solakovic $\mathrm{S}$, Mujanovic E, et al. Comparison of transperitoneal and retroperitoneal approach in aorto iliac occlusive disease. Med Arh. 2013;67:249-51.

25. Leville C, Kashyap V, Clair D, Bena J, Lyden S, Greenberg $\mathrm{R}$, et al. Endovascular management of iliac artery occlusions: Extending treatment to TransAtlantic Inter-Society Consensus class C and D patients. J Vasc Surg. 2006;43:32-9.

26. Jongkind V, Akkersdijk G, Yeung K, Wisselink W. A systematic review of endovascular treatment of extensive aortoiliac occlusive disease. J Vasc Surg. 2010;52:1376-83.

27. Indes J, Pfaff M, Farrokhyar F, Brown H, Hashim P, Cheung $\mathrm{K}$, et al. Clinical outcomes of 5358 patients undergoing direct open bypass or endovascular treatment for aortoiliac occlusive disease: A systematic review and meta-analysis. J Endovasc Ther. 2013;20:443-55.

Correspondencia: Jaime José Díaz, MD

Correo electrónico.jdiazdehoyos@gmail.com Cartagena, Colombia 